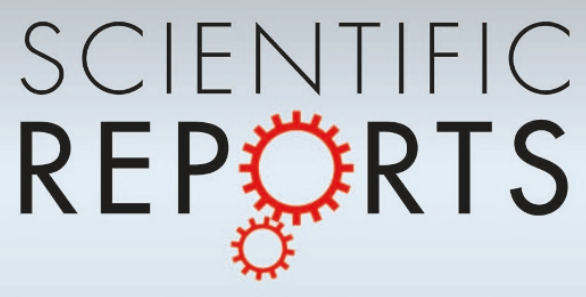

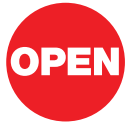

SUBJECT AREAS:

STATISTICAL PHYSICS, THERMODYNAMICS AND NONLINEAR DYNAMICS

THEORY

MODELLING AND THEORY

COMPUTATIONAL BIOLOGY

Received

9 August 2012

Accepted

28 August 2012

Published

10 September 2012

Correspondence and requests for materials should be addressed to N.M. (masuda@mist.i. u-tokyo.ac.jp)

\section{Evolution of cooperation driven by zealots}

\author{
Naoki Masuda
}

Department of Mathematical Informatics, The University of Tokyo, 7-3-1 Hongo, Bunkyo, Tokyo 113-8656, Japan.

Recent experimental results with humans involved in social dilemma games suggest that cooperation may be a contagious phenomenon and that the selection pressure operating on evolutionary dynamics (i.e., mimicry) is relatively weak. I propose an evolutionary dynamics model that links these experimental findings and evolution of cooperation. By assuming a small fraction of (imperfect) zealous cooperators, I show that a large fraction of cooperation emerges in evolutionary dynamics of social dilemma games. Even if defection is more lucrative than cooperation for most individuals, they often mimic cooperation of fellows unless the selection pressure is very strong. Then, zealous cooperators can transform the population to be even fully cooperative under standard evolutionary dynamics.

(s)

umans often behave cooperatively in social dilemma situations in which withholding cooperative behavior is logically better. In fact, cooperation in social dilemma games has been explained by various mechanisms including kin selection, assortative interactions, group competition, direct reciprocity (i.e., repeated interactions), reputation-based indirect reciprocity, and spatial or network structure of populations ${ }^{1,2}$.

In contrast, recent evidence suggests that cooperation at a population level may occur as cascading in a social network ${ }^{3}$. Such a contagious view of cooperation is distinct from the mechanisms governing cooperation explained above, which assume that individuals at least try to maximize material payoffs, a central assumption in game theory including evolutionary game theory. In contagion, individuals may imitate others' behavior without much caring the material payoff to the self and others.

The comtemporary results of the so-called upstream reciprocity (also called generalized exchange and pay-itforward reciprocity; it is a type of indirect reciprocity) are also consistent with contagious scenarios of cooperation. Upstream reciprocity, in which an individual helped by somebody helps somebody else, is widely observed in humans ${ }^{4-8}$. However, theory assuming payoff maximization does not support upstream reciprocity on its own ${ }^{9,10}$. Cooperation on the basis of upstream reciprocity is stable only in combination with a different mechanism such as direct reciprocity ${ }^{11}$, mobility of players across group ${ }^{12}$, assortative interaction ${ }^{13}$, sufficiently frequent ingroup interaction $^{14}$, and network reciprocity ${ }^{11,15,16}$.

Contagion of suboptimal behavior or attitudes seems to be even more common outside social dilemma games. Even if individuals do not like the behavior or social norm (e.g., binge drinking in colleges) and are not forced to adopt it, they often obey others. In sociology, such a phenomenon is interpreted under the framework of herd behavior, pluralistic ignorance, and false enforcement ${ }^{17,18}$.

Contagion implies that individuals change behavior under null or weak selection pressure. Consistent with this, the selection pressure for humans playing the prisoner's dilemma game was recently shown to be relatively weak; subjects did not take the imitate-the-best behavior with a probability of $\approx 30 \%{ }^{19}$ (also see [20]).

Nevertheless, even under weak selection pressure, unconditional defection remains the unique Nash equilibrium of the social dilemma game. Then, how can we explain cascades of cooperation found in experiments?

In mimicry-guided opinion formation models in which the two competing opinions are equally strong, a small number of zealot voters can attract nonzealous players to the preferred opinion of the zealot ${ }^{21-25}$. Motivated by these studies, I show that a small fraction of zealous cooperators can reliably induce cooperation at a population level. It should be noted that opinion formation models and (social dilemma) games are fundamentally different in that only the latter involves strategic interactions and natural selection. Examples of situations in which zealous cooperators in social dilemmas are witnessed include military services and team sports ${ }^{26}$, and perhaps charity campaigns. In the proposed mechanism, weak selection promotes cooperation. However, the selection pressure does not have to be as weak as assumed in other theories of cooperation (e.g., $[27,28])$. The proposed mechanism does not require additional model components such as the conformity bias ${ }^{29,30}$ or so-called cooperation facilitators that increment payoffs to cooperators, but not to defectors ${ }^{31}$. 
(a)

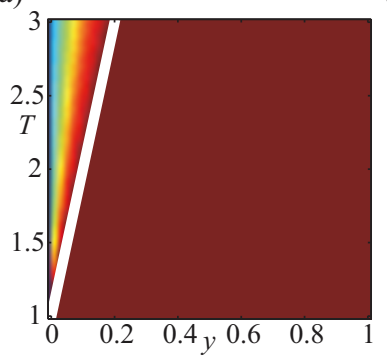

(b)

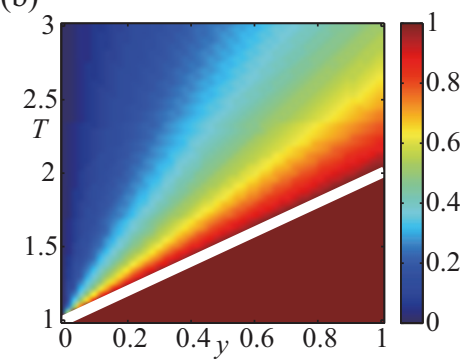

Figure 1 Fraction of cooperators among the ordinary players in the presence of perfectly zealous cooperators (i.e., $p=1$ ). The lines represent $T=1+y / w$. I used a typical payoff matrix of the prisoner's dilemma game given by $R=1, T>1$, and $S=P=0$. I set (a) $w=0.1$ and (b) $w=1$.

\section{Results}

I analyze the evolutionary dynamics given by Eqs. (8) and (9). I mainly examine the prisoner's dilemma game described by a standard payoff matrix given by $R=1, T>1$, and $S=P=0$. When zealous players are absent (i.e., $y=0$ ), $\mathrm{D}$ is the only Nash equilibrium (i.e., $x_{\mathrm{C}}=0$ ). I explore the possibility that cooperation is stabilized among ordinary players (i.e., nonzealous players) in the presence of zealous players.

Prisoners' dilemma with perfect zealous cooperators. In this section, I consider the case in which the zealous players always cooperate (i.e., $p=1, y_{\mathrm{C}}=y$ ). In this case, Eq. (8) is reduced to

$$
\frac{\mathrm{d} x_{\mathrm{C}}}{\mathrm{d} t}=\frac{1-x_{\mathrm{C}}}{\langle\pi\rangle}\left\{(1-w) y+w \frac{\left(x_{\mathrm{C}}+y\right)\left(x_{\mathrm{C}}+y-T x_{\mathrm{C}}\right)}{1+y}\right\} .
$$

Because the coefficient of $x_{\mathrm{C}}^{2}$ in $f\left(x_{\mathrm{C}}\right) \equiv(1-w) y+w\left(x_{\mathrm{C}}+y\right)\left(x_{\mathrm{C}}+y\right.$ $\left.-T x_{\mathrm{C}}\right) /(1+y)$ is negative and $f(0)=(1-w) y+w y^{2} /(1+y)>0$, the dynamics has at most one internal equilibrium, which is stable if it exists. When $f(1)>0$, i.e.,

$$
T \leq 1+\frac{y}{w},
$$

$d x_{\mathrm{C}} / d t>0\left(0 \leq x_{\mathrm{C}}<1\right)$ holds true such that the only equilibrium is located at $x_{\mathrm{C}}^{*}=1$. Then, all the players eventually cooperate. Equation (2) indicates that weak selection and the presence of many zealous players facilitate such full cooperation. If $T>1+y / w$, the stable equilibrium $x_{\mathrm{C}}^{*}$ is given by

$$
x_{\mathrm{C}}^{*}=\frac{-w y(T-2)+\sqrt{w^{2} y^{2}(T-2)^{2}+4 w(T-1) y(y+1-w)}}{2 w(T-1)} .
$$

It should be noted that $0<x_{\mathrm{C}}^{*}<1$.

The equilibrium fraction of cooperators among the ordinary players is shown as a function of $y$ and $T$ in the case of relatively weak $(w=0.1)$ and strong $(w=1)$ selection in Figs. 1(a) and 1(b), respectively. The lines represent $T=1+y / w$ and separate the full cooperation phase and the partial cooperation phase. Figure 1 indicates that the fraction of cooperators is mainly determined by $y / w$ and is larger with the weak selection than the strong selection.

Prisoner's dilemma with imperfect zealous cooperators. In fact, zealous players may not perfectly cooperate. Therefore, I investigate evolutionary dynamics given by Eqs. (8) and (9) with $p<1$ by numerically integrating them. I use the Euler-Maruyama integration scheme with $d t=0.01$. For a fixed set of parameter values, I started the evolutionary dynamics from various initial conditions, i.e., $\left(x_{\mathrm{C}}, y_{\mathrm{C}}\right)$ $=(0.05 i, 0.05 j)$, where $i$ and $j$ are integers and $1 \leq i, j \leq 19$, and confirmed that the equilibrium is independent of the initial condition.

The equilibrium fraction of cooperators among the ordinary players is shown for $(w, T)=(0.1,1.5),(0.1,2.5),(1,1.5)$, and

(a)

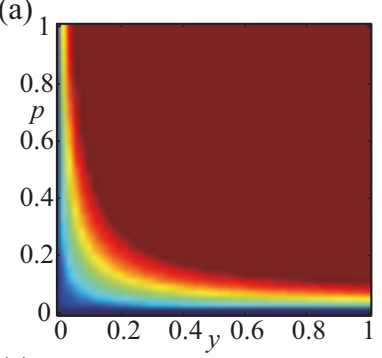

(c)
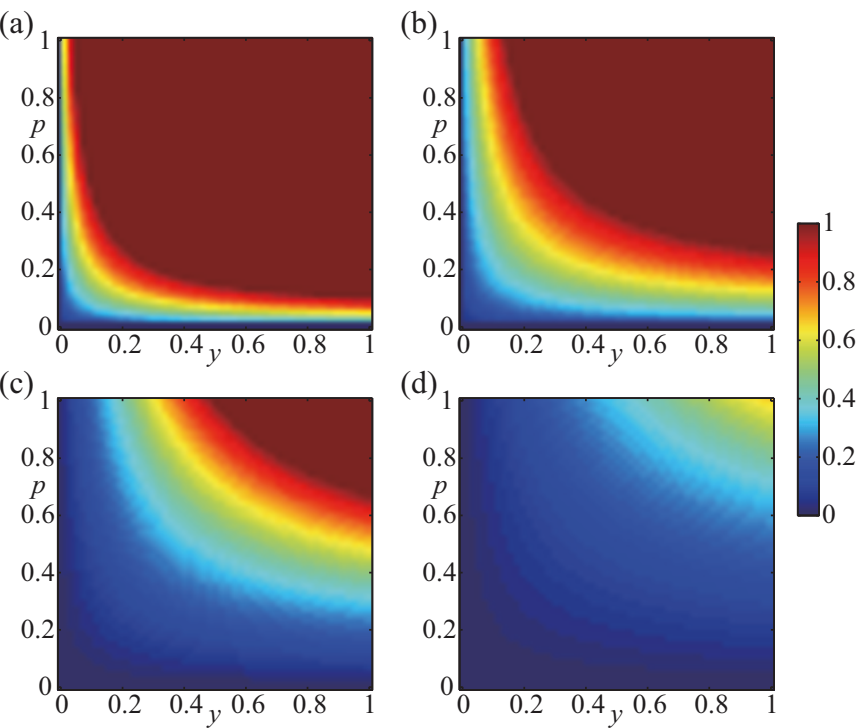

Figure $2 \mid$ Fraction of cooperators among the ordinary players as a function of the additional density of zealous players (i.e., $y$ ) and the probability of unconditional cooperation for zealous players (i.e., $p$ ). I set $R=1$ and $S=P=0$. (a) $w=0.1, T=1.5$. (b) $w=0.1, T=2.5$. (c) $w=1$, $T=1.5$. (d) $w=1, T=2.5$.

(1, 2.5) in Figs. 2(a) 2(b), 2(c), 2(d), respectively. When $w=0.1$, nearly perfect cooperation is obtained unless the fraction of zealous players (i.e., $y$ ) and the probability that zealous players unconditionally cooperate (i.e., $p$ ) are both small. This is also the case when the temptation payoff $T$ is rather large (Fig. 2(b); $T=2.5$ ). Even when $w$ $=1$, a considerable fraction of cooperation (e.g., 0.4 ) is observed for a wide parameter range when $T=1.5$ (Fig. 2(c)).

To confirm that the results are not specific to the Moran type of the reproduction process, I also implemented the so-called pairwise

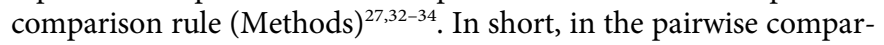
ison rule, the probability of the strategy replacement is a sigmoid function of the difference between the fitness of two randomly selected players. This update rule is implicated in recent laboratory experiments ${ }^{19}$. Numerical results for the prisoner's dilemma game with $T=1.5$ and $T=2.5$ under the pairwise comparison rule with $\bar{\beta}=0.5$ are shown in Figs. 3(a) and 3(b), respectively. The results are qualitatively the same as those under the Moran process (Fig. 2). In the rest of the present paper, I use the Moran process.

The fraction of cooperators among the ordinary players for various values of $T$ is shown in Fig. 4 . I used two large values of $p(p=0.9$ and $p=1)$ and two small values of $y(y=0.05$ and $y=0.1)$. Under both weak selection (Fig. 4(a); $w=0.1$ ) and strong selection (Fig. 4(b); $w=1$ ), the results do not depend much on the value of $p$ for large $p$. This behavior is also evident in Fig. 2. Therefore, the theoretical results obtained in the previous section for the case of perfectly cooperating zealous players (i.e., $p=1$ ) are translated to the case of imperfect zealots (i.e., $p<1$ ) without much change. In contrast, the fraction of cooperation is sensitive to the density of zealots (i.e., $y)$.

Snowdrift game. The emergence of cooperation owing to the combination of ordinary players and zealots is not restricted to the case of the prisoner's dilemma. In this section, I briefly examine the snowdrift game, also known as the chicken game and the hawk-dove game $^{35-37}$. The payoff matrix of a standard snowdrift game is given by $R=\beta-0.5, T=\beta, S=\beta-1$, and $P=0$, where $\beta>1^{37}$. I set $\beta=1.5$ such that the stable fraction of $C$ in the absence of zealots is given by $(2 \beta-2) /(2 \beta-1)=0.5$. 
(a)

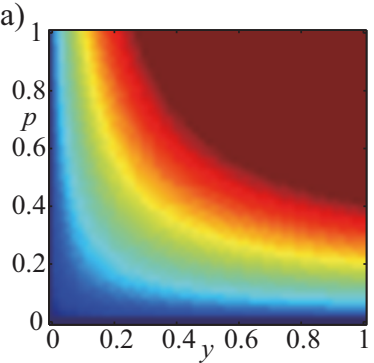

(b)

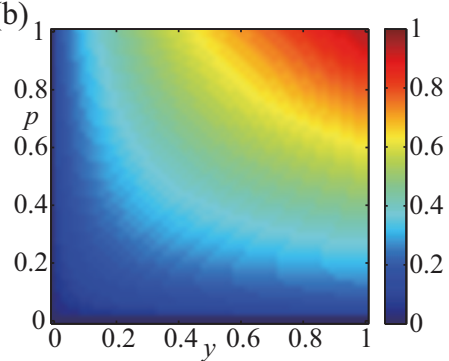

Figure 3 Fraction of cooperators among the ordinary players in the prisoner's dilemma game when the pairwise comparison rule is used for the updating. I set $R=1, S=P=0$, and $\bar{\beta}=0.5$. (a) $T=1.5$. (b) $T=2.5$.

The fraction of cooperation among the ordinary players is shown for $w=0.1$ and $w=1$ in Figs. 5(a) and 5(b), respectively. Figure 5 indicates that the fraction of cooperators is much larger than 0.5 in a wide parameter range, particularly when $w=0.1$.

\section{Discussion}

I showed that a small fraction of zealous cooperators can guide cooperation of players that obey a standard evolutionary dynamics. The numerical results indicate that the zealous players do not have to be perfectly zealous cooperators. The proposed mechanism operates better when the selection pressure is weak and the density of zealous players is large. Although I used the Moran process and the replicator dynamics, the results do not qualitatively change if a different strategy update rule called the pairwise comparison rule is used.

All the present results are independent of the initial condition. Therefore, if the dynamics is initiated from a small density of cooperation, perhaps only among zealots, cooperation can cascade to prevail in the population. I emphasize that the cascade can occur even if most players prefer defection to cooperation to some extent. My results may provide theoretical underpinning of cascades of cooperation $^{3}$ and upstream reciprocity ${ }^{4-8}$ observed in human subjects. In contrast, the emergence and maintenance of cooperation based on conformity bias ${ }^{29}$ requires that a majority of players initially cooperates.

Crucial assumptions underlying the proposed explanation of cooperation are the stochasticity of the dynamics and weak selection (i.e., small $w$ ). Weak selection is often employed in theoretical studies because, among other things, Taylor expansion on $w$ often leads to analytical conditions for cooperation (e.g., $[27,28])$. However, experiments with human subjects present evidence against excessively weak selection ${ }^{19}$. I referred to the intensity of selection equal to $w=0.1$ as weak selection. This value of $w$ may not be too small to violate the reality. In general, the effective intensity of selection depends on the payoff matrix and the strategy update rule as well

(a)

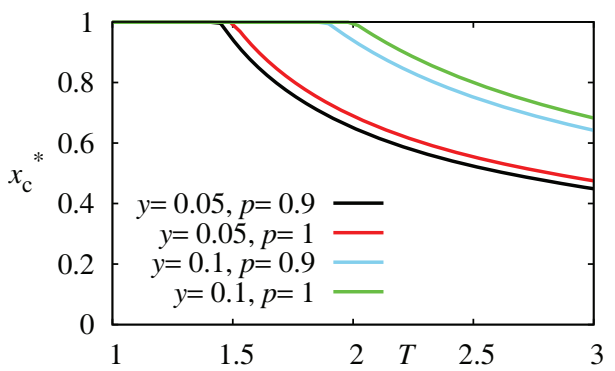

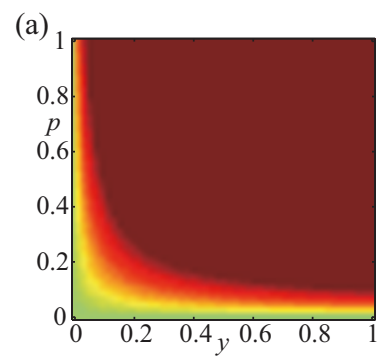

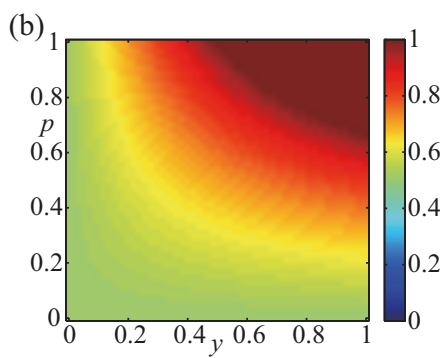

Figure $5 \mid$ Fraction of cooperators among the ordinary players as a function of $y$ and $p$ in the snowdrift game. I set $R=\beta-0.5, S=\beta-1$, $T=\beta, P=0$, and $\beta=0.5$. (a) $w=0.1$. (b) $w=1$.

on the $w$ value. Nevertheless, the following simple calculus may help: the largest and smallest possible fitness values in the prisoner's dilemma used in this study are equal to $1-w+w T$ and $1-w$, respectively. Therefore, $w=0.1$ indicates that the ratio of the two fitness values for $T=2$, for example, is equal to $(1-w+w T) /(1-$ $w)=11 / 9$. If this ratio should exceed $2, w>1 / 3$ is required under $T$ $=2$. Although I only examined the extreme two cases, i.e., $w=0.1$ and $w=1$, the results shown in the figures imply that much cooperation will be observed with $w=1 / 3$. In fact, some cooperation is observed even with $w=1$ if proper conditions are met (Figs. 1(b) and $2(c))$.

It should be noted that I assumed that players, either ordinary or zealous, have the same strength of influence on others. Although the heterogeneity in the influence of individuals, i.e., power, would shape collective behavior of humans, the present contribution is not about the power but about the relationship between zealots, weak selection, contagion, and cooperation.

I did not ask the origin of zealous cooperators. Trivially, they will not emerge as a result of evolution unless other games or dynamics are simultaneously considered. One interpretation of this assumption is that zealous players are not interested in maximizing the material payoff. Zealous cooperators are found in some real situations ${ }^{26}$. Another interpretation is that zealots are payoff maximizers but have different payoff functions. In theory of collective action, heterogeneity in interests and resources of individuals are suggested to elicit collective action to solve the free rider problem (see [38] for a review). Although the present mechanism is independent of that of collective action, zealots may perceive payoffs differently from ordinary players such that cooperation may not incur social dilemma for zealots.

\section{Methods}

Model. I consider evolutionary dynamics of an infinite well-mixed population in which each pair of players is involved in the symmetric two-player two-strategy game once per generation. The payoff matrix is defined by

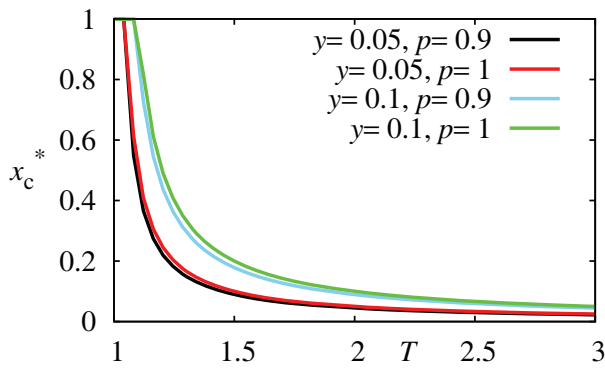

Figure $4 \mid$ Fraction of cooperators among the ordinary players as a function of the temptation payoff (i.e., $T$ ). I set $R=1$ and $S=P=0$. (a) $w=0.1$. (b) $w=1$. 


$$
\begin{aligned}
& \text { C D } \\
& \text { D }\left(\begin{array}{ll}
R & S \\
T & P
\end{array}\right) \text {, }
\end{aligned}
$$

where the entries of Eq. (4) represent the payoffs that the row player gains. Each row (column) corresponds to the action of the row (column) player, i.e., cooperation (C) or defection (D).

I assume two types of players. A player of the first type, called ordinary player, obeys a standard evolutionary dynamics described below. A player of the second type, called zealous player, may obey the evolutionary dynamics or unconditionally cooperate.

The evolutionary dynamics is defined as follows. The summation of the payoff over all the opponents defines the aggregated payoff to a player. The fitness, i.e., the propensity to reproduce, of a player is a linear function of the payoff. The proportionality constant controls the intensity of selection. At the end of each generation, a single player whose strategy (i.e., C or D) is replaced is selected with the equal probability from the population. If the selected player is ordinary player, a parent, which is either ordinary or zealous player, is selected from the entire population with the probability proportional to the fitness. Then, the strategy of the updated player is replaced by that of the parent player. This update process is equivalent to the Moran process, a standard model of the birth-death process (e.g., [2]). If the updated player is zealous player, its strategy turns to $C$ with probability $p$. With probability $1-p$, the updated player obeys the rule used by the ordinary player to adopt the strategy of a parent selected with the probability proportional to the fitness.

I normalize the density of the ordinary players to unity and denote the added density of zealous players by $y(\geq 0)$. The densities of cooperators among the ordinary and zealous players are denoted by $x_{\mathrm{C}}\left(0 \leq x_{\mathrm{C}} \leq 1\right)$ and $y_{\mathrm{C}}\left(0 \leq y_{\mathrm{C}} \leq y\right)$, respectively. The mean fitness, with the divisive factor $1+y$ (i.e., the total population density) intentionally neglected, is defined by

$$
\langle\pi\rangle \equiv\left(x_{\mathrm{C}}+y_{\mathrm{C}}\right) \pi_{\mathrm{C}}+\left(1-x_{\mathrm{C}}+y-y_{\mathrm{C}}\right) \pi_{\mathrm{D}},
$$

where

$$
\pi_{\mathrm{C}}=1-w+\frac{w\left[\left(x_{\mathrm{C}}+y_{\mathrm{C}}\right) R+\left(1-x_{\mathrm{C}}+y-y_{\mathrm{C}}\right) S\right]}{1+y}
$$

and

$$
\pi_{\mathrm{D}}=1-w+\frac{w\left[\left(x_{\mathrm{C}}+y_{\mathrm{C}}\right) T+\left(1-x_{\mathrm{C}}+y-y_{\mathrm{C}}\right) P\right]}{1+y}
$$

are the fitness to a $\mathrm{C}$ and $\mathrm{D}$ player, respectively, and $w(0 \leq w \leq 1)$ indicates the intensity of selection ${ }^{27,28}$. Equations (6) and (7) indicate that the payoff to a player per opponent is translated to the fitness with proportionality constant $w$. I assume $\langle\pi\rangle>0$ such that the selection of the parent player with the probability proportional to the fitness is well defined.

In the reproduction phase, a cooperator and defector are selected as parent with probability $\left(x_{\mathrm{C}}+y_{\mathrm{C}}\right) \pi_{\mathrm{C}} /\langle\pi\rangle$ and $\left(1-x_{\mathrm{C}}+y-y_{\mathrm{C}}\right) \pi_{\mathrm{D}} /\langle\pi\rangle$, respectively. Therefore, the dynamics of the fraction of cooperators among the ordinary players is given by

$$
\frac{\mathrm{d} x_{\mathrm{C}}}{\mathrm{d} t}=\frac{1}{\langle\pi\rangle}\left[\left(x_{\mathrm{C}}+y_{\mathrm{C}}\right) \pi_{\mathrm{C}}\left(1-x_{\mathrm{C}}\right)-\left(1-x_{\mathrm{C}}+y-y_{\mathrm{C}}\right) \pi_{\mathrm{D}} x_{\mathrm{C}}\right] .
$$

When $y=0$, Eq. (8) is equivalent to the meanfield equation of the Moran process. If the divisive factor $\langle\pi\rangle$, which just controls the time scale of the dynamics in this special case, is neglected, Eq. (8) is reduced to the usual replicator dynamics.

The dynamics of the density of cooperators among the zealous players is given by

$$
\frac{\mathrm{d} y_{\mathrm{C}}}{\mathrm{d} t}=p\left(y-y_{\mathrm{C}}\right)+\frac{1-p}{\langle\pi\rangle}\left[\left(x_{\mathrm{C}}+y_{\mathrm{C}}\right) \pi_{\mathrm{C}}\left(y-y_{\mathrm{C}}\right)-\left(1-x_{\mathrm{C}}+y-y_{\mathrm{C}}\right) \pi_{\mathrm{D}} y_{\mathrm{C}}\right] .
$$

When $p=1$, zealous players always cooperate (i.e., $y_{\mathrm{C}}=y$ ). In this case, Eq. (8), with $\langle\pi\rangle$ in the denominator neglected, is equivalent to Example 2 given in [39].

Pairwise comparison rule. In the so-called pairwise comparison rule, the probability that the replacement occurs depends on the difference between the payoffs to two randomly selected players. At the end of each generation, I randomly select two players from the population without bias. If the two players are both cooperators or both defectors, nothing takes place. Otherwise, $\mathrm{C}$ replaces $\mathrm{D}$ with probability $1 /\left[1+e^{-\bar{\beta}\left(\pi_{\mathrm{C}}-\pi_{\mathrm{D}}\right)}\right]$, and $\mathrm{D}$ replaces $\mathrm{C}$ with probability $1-1 /\left[1+e^{-\bar{\beta}\left(\pi_{\mathrm{C}}-\pi_{\mathrm{D}}\right)}\right]=1 /\left[1+e^{-\bar{\beta}\left(\pi_{\mathrm{D}}-\pi_{\mathrm{C}}\right)}\right]^{27,32-34}$. The intensity of selection is controlled by $\bar{\beta}(\geq 0)$.

The evolutionary dynamics for the infinite population under the pairwise comparison rule is represented by

$$
\frac{\mathrm{d} x_{\mathrm{C}}}{\mathrm{d} t}=\frac{2}{1+y}\left[\frac{\left(1-x_{\mathrm{C}}\right)\left(x_{\mathrm{C}}+y_{\mathrm{C}}\right)}{1+e^{-\bar{\beta}\left(\pi_{\mathrm{C}}-\pi_{\mathrm{D}}\right)}}-\frac{x_{\mathrm{C}}\left(1-x_{\mathrm{C}}+y-y_{\mathrm{C}}\right)}{1+e^{-\bar{\beta}\left(\pi_{\mathrm{D}}-\pi_{\mathrm{C}}\right)}}\right]
$$

$$
\frac{\mathrm{d} y_{\mathrm{C}}}{\mathrm{d} t}=p\left(y-y_{\mathrm{C}}\right)+\frac{2(1-p)}{1+y}\left[\frac{\left(y-y_{\mathrm{C}}\right)\left(x_{\mathrm{C}}+y_{\mathrm{C}}\right)}{1+e^{-\bar{\beta}\left(\pi_{\mathrm{C}}-\pi_{\mathrm{D}}\right)}}-\frac{y_{\mathrm{C}}\left(1-x_{\mathrm{C}}+y-y_{\mathrm{C}}\right)}{1+e^{-\bar{\beta}\left(\pi_{\mathrm{D}}-\pi_{\mathrm{C}}\right)}}\right] .
$$

1. Nowak, M. A. Five rules for the evolution of cooperation. Science 314, 1560-1563 (2006).

2. Nowak, M. A. Evolutionary Dynamics (The Belknap Press of Harvard University Press, MA, 2006).

3. Fowler, J. H. \& Christakis, N. A. Cooperative behavior cascades in human social networks. Proc. Natl. Acad. Sci. USA 107, 5334-5338 (2010).

4. Yamagishi, T. \& Cook, K. S. Generalized exchange and social dilemmas. Social Psychology Quarterly 56, 235-248 (1993).

5. Molm, L. D., Collett, J. L. \& Schaefer, D. R. Building solidarity through generalized exchange: a theory of reciprocity. Am. J. Sociol. 113, 205-242 (2007).

6. Dufwenberg, M., Gneezy, U., Güth, W. \& van Damme, E. Direct vs indirect reciprocity: an experiment. Homo Oecono. 18, 19-30 (2001).

7. Greiner, B. \& Levati, M. V. Indirect reciprocity in cyclical networks-an experimental study. J. Econ. Psych. 26, 711-731 (2005).

8. Stanca, L. Measuring indirect reciprocity: whose back do we scratch? J. Econ. Psych. 30, 190-202 (2009).

9. Boyd, R. \& Richerson, P. J. The evolution of indirect reciprocity. Soc. Netw. 11, 213-236 (1989).

10. Masuda, N. Clustering in large networks does not promote upstream reciprocity. PLOS ONE 6, e25190 (2011).

11. Nowak, M. A. \& Roch, S. Upstream reciprocity and the evolution of gratitude. Proc. R. Soc. Lond. B 274, 605-610 (2007).

12. Hamilton, I. M. \& Taborsky, M. Contingent movement and cooperation evolve under generalized reciprocity. Proc. R. Soc. B 272, 2259-2267 (2005).

13. Rankin, D. J. \& Taborsky, M. Assortment and the evolution of generalized reciprocity. Evolution 63, 1913-1922 (2009).

14. Barta, Z., McNamara, J. M., Huszár, D. B. \& Taborsky, M. Cooperation among nonrelatives evolves by state-dependent generalized reciprocity. Proc. R. Soc. B 278, 843-848 (2011).

15. Iwagami, A. \& Masuda, N. Upstream reciprocity in heterogeneous networks J. Theor. Biol. 265, 297-305 (2010).

16. van Doorn, G. S. \& Taborsky, M. The evolution of generalized reciprocity on social interaction networks. Evolution 66, 651-664 (2012).

17. Centola, D., Willer, R. \& Macy, M. The Emperor's Dilemma: A computational model of self-enforcing norms. Am. J. Sociol. 110, 1009-1040 (2005)

18. Willer, R., Kuwabara, K. \& Macy, M. W. The false enforcement of unpopular norms. Am. J. Sociol. 115, 451-490 (2009).

19. Traulsen, A., Semmann, D., Sommerfeld, R. D., Krambeck, H. J. \& Milinski, M. Human strategy updating in evolutionary games. Proc. Natl. Acad. Sci. USA 107, 2962-2966 (2010).

20. Kirchkamp, O. \& Nagel, R. Naive learning and cooperation in network experiments. Games Econ. Behav. 58, 269-292 (2007).

21. Mobilia, M. Does a single zealot affect an infinite group of voters? Phys. Rev. Lett. 91, 028701 (2003).

22. Mobilia, M., Petersen, A. \& Redner, S. On the role of zealotry in the voter model. J. Stat. Mech. P08029 (2007).

23. Galam, S. \& Jacobs, F. The role of inflexible minorities in the breaking of democratic opinion dynamics. Physica A 381, 366-376 (2007).

24. Xie, J. et al. Social consensus through the influence of committed minorities. Phys. Rev. E 84, 011130 (2011).

25. Singh, P., Sreenivasan, S., Szymanski, B. K. \& Korniss, G. Accelerating consensu on coevolving networks: The effect of committed individuals. Phys. Rev. E $\mathbf{8 5}$ 046104 (2012).

26. Coleman, J. S. Free riders and zealots: The role of social networks. Sociol. Th. 6 , 52-57 (1988).

27. Nowak, M. A., Sasaki, A., Taylor, C. \& Fudenberg, D. Emergence of cooperation and evolutionary stability in finite populations. Nature 428, 646-650 (2004).

28. Ohtsuki, H., Hauert, C., Lieberman, E. \& Nowak, M. A. A simple rule for the evolution of cooperation on graphs and social networks. Nature 441, 502-505 (2006).

29. Boyd, R. \& Richerson, P. J. Culture and the Evolutionary Process (The University of Chicago Press, Chicago, 1985).

30. Szolnoki, A., Wang, Z. \& Perc, M. Wisdom of groups promotes cooperation in evolutionary social dilemmas. Sci. Rep. 2, 576 (2012).

31. Mobilia, M. Stochastic dynamics of the prisoner's dilemma with cooperation facilitators. Phys. Rev. E 86, 011134 (2012).

32. Blume, L. The statistical mechanics of strategic interaction. Games Econ. Behav. 5, 387-424 (1993).

33. Szabó, G. \& Tőke, C. Evolutionary prisoner's dilemma game on a square lattice. Phys. Rev. E 58, 69-73 (1998).

34. Traulsen, A., Nowak, M. A. \& Pacheco, J. M. Stochastic dynamics of invasion and fixation. Phys. Rev. E 74, 011909 (2006).

35. Maynard Smith, J. Evolution and the Theory of Games (Cambridge University Press, Cambridge, UK, 1982)

36. Sugden, R. The Economics of Rights, Co-operation and Welfare (Blackwell, New York, NY, 1986). 
37. Hauert, C. \& Doebeli, M. Spatial structure often inhibits the evolution of cooperation in the snowdrift game. Nature 428, 643-646 (2004).

38. Oliver, P. E. Formal models of collective action. Annu. Rev. Sociol. 19, 271-300 (1993).

39. Chatterjee, K., Zufferey, D. \& Nowak, M. A. Evolutionary game dynamics in populations with different learners. J. Theor. Biol. 301, 161-173 (2012).

\section{Acknowledgments}

I thank Mitsuhiro Nakamura and Shoma Tanabe for careful reading of the manuscript and Masanori Takezawa for valuable discussions. This work is supported by Grants-in-Aid for
Scientific Research (Nos. 20760258 and 23681033, and Innovative Areas "Systems Molecular Ethology" (No. 20115009)) from MEXT, Japan.

\section{Additional information}

Competing financial interests: The author declares no competing financial interests.

License: This work is licensed under a Creative Commons

Attribution-NonCommercial-ShareAlike 3.0 Unported License. To view a copy of this license, visit http://creativecommons.org/licenses/by-nc-sa/3.0/

How to cite this article: Masuda, N. Evolution of cooperation driven by zealots. Sci. Rep. 2 , 646; DOI:10.1038/srep00646 (2012). 